\title{
Effects of retention interval and modality on sensory and semantic trace information
}

\author{
DOUGLAS L. NELSON and CATHY L. McEVOY \\ University of South Florida, Tampa, Florida 33620
}

\begin{abstract}
Subjects studied a long list of individual words that were presented either visually or auditorily. Recall was tested immediately or after a filled delay by using either word endings or taxonomic categories as extralist retrieval cues. Two interactions were of particular interest. First, word ending cues were just as effective as taxonomic cues on the immediate test. On the delayed test, however, ending cues were less effective. This result suggests that sensory information encoded about a word decays at a faster rate than semantic information. Second, although modality had no observable influence on the taxonomic cues, word ending cues were more effective when all items were shown visually than when they were presented auditorily. Taken together, these findings indicate that the visual features of words are encoded at study and that this information can be accessed during test if it is recapitulated by the retrieval cue shortly after acquisition.
\end{abstract}

Words and pictures are theoretically represented in memory in terms of their associated visual, phonemic, and semantic features. These features are conceptualized as being independent and as representing qualitatively different "types" (Nelson, 1979) or "levels" (Craik \& Lockhart, 1972) of information. Moreover, it now seems apparent that semantically emphasized encodings prompted by semantic cues are more likely to be recalled than sensory emphasized encodings prompted by sensory cues (e.g., Craik \& Tulving, 1975; Nelson \& McEvoy, 1979; Nelson, Walling, \& McEvoy, 1979). This semantic superiority effect is usually explained by assuming that semantic encodings are more distinctive (Craik, 1979) or more interactive (Nelson, 1979; Nelson \& McEvoy, 1979). However, as Craik and Lockhart (1972) have suggested, semantic superiority may arise because sensory encodings decay at relatively faster rates. Unfortunately, the evidence on this point is mixed, with several studies reporting equivalent rates (Bregman, 1968; Jacoby, 1975; Shulman, 1970) and others suggesting faster rates (Cermak \& Youtz, 1976; Kintsch \& Buschke, 1969). Thus, the question of differential decay is unresolved.

These apparently conflicting results may be related to procedural differences. They may also be related to one or more of four other considerations. First, preexperimental associative similarities between the retrieval cues and their targets were not controlled. Like associates, rhymes vary in strength. It would be no surprise to discover that strong associates can be more effective cues than weak rhymes (Nelson, Wheeler, Borden, \& Brooks, 1974). Second, the number of relevant alterna-

This research was supported by Grant MH 16360 to the first author from the National Institute of Mental Health. Requests for reprints should be sent to Douglas Nelson, Department of Psychology, University of South Florida, Tampa, Florida 33620. tives associated to each of the cues was not controlled. Recall is inversely related to set size, and there are systematic interactions between this factor and type of cue (Nelson \& McEvoy, 1979). Third, no attempts were made to control initial learning prior to assessing decay rate (Underwood, 1964). Finally, as Cermak and Youtz (1976) have noted, apparent differences in decay rates may be reflecting differential vulnerability to interference when retention interval is manipulated by varying the number of intervening items. One purpose of the present experiment was to compare the relative effectiveness of sensory and semantic cues on an immediate test and after a filled delay. Cue-target associative similarity and set size were manipulated, and encoding conditions were selected to minimize any differences between the two types of cues on the immediate test. Moreover, the delay task was very different from the word encoding task.

Target words were shown individually during study under intentional learning conditions; during test, either word endings or taxonomic category names served as extralist retrieval cues (e.g., IME or An American Coin as cues for DIME). Previous research has indicated that these two types of cues were equally effective in these circumstances (Nelson \& McEvoy, 1979). Endings and taxonomic categories were used instead of rhymes and associates in order to minimize interference within the irrelevant dimension; for example, rhymes apparently generate a form of semantic interference (Nelson et al., 1979).

Based on previous findings, recall on the immediate test was expected to be higher for strong cues and for small sets. It was not expected to vary with cue type. Recall on the delayed test was expected to be dependent upon the relative decay rates for the two types of cues. Equivalent cue effectiveness on the delay test would suggest that the differences between these two types of 
cues, when they are obtained, would be better explained by resorting to some principle other than decay. $\mathrm{Al}$ ternatively, any interactions between cue type and delay, including higher order interactions with strength or set size, would suggest that at least some of the semantic superiority effect should be attributed to the greater transience of sensory traces.

The second purpose of the present study was to isolate the relative contributions of visual and phonemic features to the effectiveness of the word ending cues. If visual information linked to a particular word is encoded and maintained (Kolers, 1976; Light \& Berger, 1976; Nelson, Brooks, \& Borden, 1974), then these ending cues should be more effective when items are presented visually rather than auditorily. Thus, presentation modality was manipulated in addition to cue type and delay. It should be noted that Cermak and Youtz (1976) failed to find any effect of modality on rhyme cues. However, they selected rhymes that did not share visual features, so the possibility of visual facilitation could not be evaluated. Modality was not expected to have any effect on the taxonomic cues. Semantic information encoded about an item should be functionally equivalent regardless of the form of the stimulus (Nelson, 1979; Nelson, Reed, \& McEvoy, 1977). A semantic code can be accessed from a picture or from a visually or auditorily presented stimulus. In all cases, the semantic code is thought to be functionally identical.

\section{METHOD}

\section{Design}

The experimental design was a 2 by 2 by 2 by 2 by 3 mixedmodel factorial. Type of cue, presentation modality, and retention interval were between-subjects factors. Strength of cuetarget relationship and the three levels of set size were manipulated within subjects.

\section{Materials}

The lists were identical to those used by Nelson and McEvoy (1979). Briefly, controlled association norms were collected for word endings and for taxonomic categories. For endings, subjects wrote down the first response that came to mind that rhymed with the sound just heard; for taxonomic categories, subjects wrote the first response that belonged to the category just heard. These norms were used to estimate both cue-target strength and set size. Thus, eight lists were constructed, four for each cue type. The cues had served as stimuli in the normative task and the targets were generated by different proportions of subjects in that task. Ending cues overlapped visually and phonemically with their targets.

Of the 24 pairs in each list, 12 were strongly associated and 12 were weakly associated. For strong pairs, averages of $.32(\mathrm{SD}=.07)$ and $.34(\mathrm{SD}=.10)$ of the subjects provided the targets in response to the cues in the ending and taxonomic norms. For weak pairs, these averages were $.03(.02)$ and .03 (.02). Thus, cue-target associative similarity was equated at two different levels of cue types. Within each level of strength, the 12 pairs were subdivided into three different set sizes. Set sizes were estimated from the total number of different, but appropriate, responses given to each cue in the normative task. For example, with the ending OON, a large ending category, a total of 22 different words sharing this sound was generated by a sample of 184 subjects. With small, medium, and large ending categories, averages of 5.63 (.96), 13.44 (1.41), and $25.50(5.13)$ different words were produced; for taxonomic categories, these values were $5.69(1.01), 14.13(1.26)$, and 25.38 (4.13). Thus, set sizes were matched for each cue type.

To control for the set-size factor within the strength manipulation, the cues having a strong relation to their targets in Lists 1 and 3 had a weak relation to their targets in Lists 2 and 4 , that is, ENCH served as a strong cue for BENCH in List 1 and as a weak cue for TRENCH in List 2. This procedure resulted in using the same cues for two of the four lists in each cue type. The targets, however, were different for all eight lists.

\section{Procedure}

Subjects participated in individual sessions and received a single study-test trial. During study, targets were presented at a 3.3-sec rate and, during test, the cues were presented at a subjectpaced rate. The order of presentation was unsystematically randomized on both study and test trials for every subject. A name reading task (e.g., Cathy McEvoy, etc.) served to acquaint subjects with the rate and procedure for the study trial.

All subjects were told that a long list of individual words would be presented only once and that they should concentrate on remembering as many as possible. No information about test cues was presented prior to study. Test instructions were equal in length for both cue types, requiring about $3 \mathrm{~min}$ to deliver, and they described the nature of the relation between cues and their targets. Explicit examples were provided, and guessing was strongly encouraged.

In the visual presentation conditions, targets appeared in uppercase letters and were presented by a slide projector through a screen in front of the subject. Each item had to be pronounced aloud by the subject when shown during study. Test cues were underlined, and they also had to be pronounced aloud. Any misreadings were immediately corrected. In the auditory conditions, the experimenter read the targets and cues aloud, with the subject shadowing her on each item.

Instructions for the test trial were read immediately following the last target for half of the subjects and after a $15-\mathrm{min}$ filled delay for the remaining half. Subjects in the delay conditions were told that the next phase of the experiment would be conducted in another room. Once they were seated and blindfolded, they were presented with 10 common substances found in most kitchens. With the opening of each vial, the experimenter called out a number from 1 to 10 , in sequence, as the subject sniffed its odor. Following this study trial, each vial was re-presented in an unsystematic random order and the subject was asked to respond with its number. The experimenter provided corrective feedback. Study-test practice alternated that way until a total of $11 \mathrm{~min}$ had elapsed since the showing of the last target in the word list. At that point, subjects returned to the experimental room and were read the test trial instructions. Odor learning was chosen to discourage rehearsal with an engaging task that, on face value, did not require much visual and semantic processing.

\section{Subjects}

Twelve subjects were assigned to each of the eight betweensubjects conditions, with four assigned to each list. All were selected from courses in introductory psychology under an incentive system. They were assigned in replication blocks of 32 and, within blocks, assignment to conditions was random.

\section{RESULTS}

Probabilities of correct recall for the visual presentation conditions are shown in Table 1 . The first result to note is that word ending cues (.72) and taxonomic category cues (.68) were equally effective when presented on the immediate test. This finding replicates 
Table 1

Probability of Correct Recall in the Visual Presentation Conditions as a Function of Cue Type and Delay

\begin{tabular}{lccc} 
& \multicolumn{3}{c}{ Cue Type } \\
\cline { 2 - 4 } Retention Interval & Ending & Taxonomic & Mean \\
\hline Immediate & .72 & .68 & .70 \\
Delay (15 min) & .54 & .64 & .59 \\
Delay (30 min) & .48 & .63 & .55 \\
Mean & .58 & .65 & \\
\hline
\end{tabular}

earlier results and suggests that these two types of cues were initially equivalent in providing access to the stored representations of their targets. The next result to note is shown in the last column. Delaying the test trial by 15 min reliably reduced recall $[F(1,44)=10.64]$. More importantly, this delay appeared to affect a relatively greater loss for the ending than for the taxonomic cues. However, the interaction of Delay by Cue Type failed to reach the .05 confidence level $[F(1,44)=3.91]$.

Because of the potential theoretical importance of this interaction, data were collected for two additional groups, each with 12 subjects. They were treated like the subjects receiving the 15 -min delay, except, in this instance, they had to learn number assignments for 16 odors and the total delay was extended to $30 \mathrm{~min}$. The analysis including all three delay conditions indicated that delay $[F(2,66)=9.84]$, cue type $[F(1,66)=$ 5.64], and the Delay by Cue Type interaction $[F(2,66)=3.60]$ were all reliable. The MSe for all of these sources was .015 . Thus, while ending and taxonomic category cues were equally effective on the immediate test, ending cues were relatively less effective after a filled delay. Sensory information seems to be lost at a faster rate than semantic information. Fisher's least significant difference (1.s.d.) for the interaction was .09 .

Probabilities of correct recall for presentation modality are shown in Table 2 . The statistical analysis of these data indicated that delay $[F(1,88)=18.76]$, modality $[F(1,88)=7.40]$, and cue type $[F(1,88)=12.91]$ were significant. Most importantly, the Modality by Cue Type interaction was also reliable $[F(1,88)=5.68]$. This interaction reflected the difference between the orally presented ending cues and the other cues, which is illustrated in the last row of the table. An 1.s.d. of .07

Table 2

Probability of Correct Recall as a Function of Presentation Modality, Cue Type, and Retention Interval

\begin{tabular}{|c|c|c|c|c|c|}
\hline \multirow[b]{3}{*}{$\begin{array}{l}\text { Retention } \\
\text { Interval }\end{array}$} & \multicolumn{4}{|c|}{ Presentation Modality } & \multirow[b]{3}{*}{ Mean } \\
\hline & \multicolumn{2}{|c|}{ Visual } & \multicolumn{2}{|c|}{ Auditory } & \\
\hline & $\begin{array}{l}\text { End- } \\
\text { ing }\end{array}$ & $\begin{array}{l}\text { Taxo- } \\
\text { nomic }\end{array}$ & $\begin{array}{l}\text { End- } \\
\text { ing }\end{array}$ & $\begin{array}{l}\text { Taxo- } \\
\text { nomic }\end{array}$ & \\
\hline Immediate & .72 & .68 & .54 & .72 & .67 \\
\hline Delay (15 min) & .54 & .64 & .45 & .59 & .55 \\
\hline Mean & .63 & .66 & .50 & .65 & \\
\hline
\end{tabular}

indicated that all of the other cues were reliably more effective than orally presented ending cues. Thus, oral presentation significantly reduced the effectiveness of the word ending cues. None of the other betweensubjects interactions was reliable. The MSe for all of these sources was .096 .

This statistical analysis also indicated that probability of recall was higher for strong (.75) than for weak $(.47)$ cues $[F(1,88)=192.03$, MSe $=.057]$, but this effect was qualified by a Cue Type by Strength interaction $[\mathrm{F}(1,88)=17.75, \mathrm{MSe}=.057]$. The difference between strong and weak cues was greater for ending cues (respectively, .74 and .38) than for taxonomic cues (respectively, .75 and .56). No other sources involving the strength variable were reliable. Finally, set size was the only other significant effect $[F(2,176)=$ 15.99, $\mathrm{MSe}=.050]$. Recall decreased as set size was increased from small (.68), to medium (.61), to large (.55). An 1.s.d. of .04 indicated that all of these means were different from one another. None of the remaining variables reliably interacted with set size, including strength of cue-target relationship.

The interaction of Modality by Cue Type indicated that modality had no effect on the taxonomic cues and that visually presented word endings were more effective than auditorily presented endings. The latter result suggested that visual information might be encoded during study and used during test, provided that the test is given soon enough after the study phase. It is interesting to note that recall in the visual condition after a 15 -min delay (.54) was equivalent to that obtained in the auditory condition on the immediate test (.54). Such equality might have been fortuitous, but the tempting implication is that the encoded visual features of words will become unavailable over this relatively short interval of time. However, at least one other interpretation is possible. Visual information may have been used only during the test trial itself to restrict set size by excluding relevant items that did not share both visual and phonemic features. If so, then set size would be smaller for visually presented ending cues than for auditorily presented cues. That is, visual information may have served only to functionally restrict set size at test, and it could be reasonably argued that visual information was not effectively encoded during study. Of course, this argument implies that there should have been an interaction between cue type, set size, and modality. Set-size effects should have been differentially reduced for visually presented endings, but they were $\operatorname{not}[\mathrm{F}(2,176)=1.77]$.

As a further check on this argument, immediate test trial data were collected for two more conditions. Under circumstances identical to the main experiment, targets were presented either visually or auditorily and, respectively, cues were presented either auditorily or visually. When combined with the visual-visual and the auditory-auditory conditions, this experiment formed 
a 2 by 2 factorial with modality varied both during study and during test. If visually presented endings were more effective only because they restricted set size at test, then visually presented test cues should be more effective than auditorily presented test cues, and modality during study should have no effect. Alternatively, if visual information is encoded during study and recapitulated at test by the cue, then the visual-visual condition should be superior to the other conditions, which should not differ. The results were consistent with the latter alternative. Probability of correct recall for the visual-visual condition was .72 . For auditoryvisual, visual-auditory, and auditory-auditory, the respective probabilities were $.57, .58$, and .54 . An 1.s.d. of .09 indicated that all three of these conditions differed reliably from visual-visual, but were not different from one another. Thus, visual information appeared to be encoded at study, and this information can apparently be accessed at test if it is recapitulated by the retrieval cue within a relatively short period of time.

\section{DISCUSSION}

The present results replicate and extend earlier findings (Nelson \& McEvoy, 1979). On an immediate test, visually presented word endings are as effective as taxonomic category names as extralist retrieval aids. However, the present results indicate that the effectiveness of the ending cues depends upon both modality and delay. They are reliably more efficient when all items are presented visually than when all are presented auditorily. Visual information linked to each target word is apparently encoded and is capable of being reactivated at test by an appropriate retrieval cue. However, if the test is delayed by 15 to $30 \mathrm{~min}$, visually presented endings are clearly less effective than taxonomic category names as redintegrative cues. Thus, when preexperimental associative similarity, set size, and initial learning are equated, the rate of loss for sensory features appears to be greater than the rate of loss for meaning features. Sensory traces seem to be less stable over time.

These findings suggest that at least some portion of the semantic superiority effect might be attributed to the greater apparent transience of sensory codes. As such, these findings are partially consistent with the original formulation of levels of processing as proposed by Craik and Lockhart (1972). They suggested that "there is usually no need to store the products of preliminary analyses" (p. 675), and that memory should be "viewed as a continuum from the transient products of sensory analyses to the highly durable products of semantic-associative operations" (p.676). These statements clearly suggest the assumption that sensory information should be more labile than semantic information. The present data offer support for this view. However, the data do not support the most extreme form of this assumption. It is feasible that the results of sensory analyses are not stored at all; they serve only as the stepping stone for more elaborate semantic coding. However, if this were the case, it would be hard to understand why word endings work so well as cues and, particularly, why their effectiveness differentially declines over the delay. The results of sensory analyses are apparently encoded in a representation that does not immediately fade away as other types of features are analyzed. This representation is presumably abstract, rather than literal or iconic (Light \& Berger, 1976). It persits long enough to facilitate cued recall on an immediate test, even though recall may be separated by many intervening study and test items and a 3-min instruction interval. Thus, although sensory encodings may be less persistent than semantic encodings, they seem to persist long enough to provide considerable support for retrieval on a test occurring within minutes after initial exposure.

This apparent persistence has interesting implications. The majority of studies comparing the relative effectiveness of sensory and semantic cues have been made under conditions of immediate testing and the presence of cues during both study and test (e.g., Craik \& Tulving, 1975; Nelson et al., 1974; Nelson \& McEvoy, 1979). Semantic superiority effects obtained under these conditions cannot be attributed only to relative code durability. Some of this superiority may be explained through this mechanism, but other factors appear to be important as well. However, there is some disagreement as to how these factors should be characterized.

On the one hand, Craik (Craik, 1979; Fisher \& Craik, 1977) has suggested that the depth to which a stimulus is analyzed, in conjunction with its degree of elaboration, gives rise to a trace that is more or less distinctive. Distinctiveness refers to the similarity among traces created by operations carried out during encoding, operations that are related to the depth and the degree of elaboration of stimulus analysis. In addition, Craik suggests the need for the notion of encoding/retrieval compatibility (cf., Tulving, 1979). Thus, according to this view, semantic codes are deeper, more likely to be elaborated, and, therefore, more distinctive than sensory codes. Because they are more distinctive, they are more likely to be recalled under conditions of high cue-totrace compatibility.

On the other hand, Nelson (Nelson, 1979; Nelson \& McEvoy, 1979) has suggested that semantic superiority effects, when they are obtained, are related more to cue-target interactiveness and perceived cue-to-trace similarity. Nelson has suggested that semantic encodings are more interactive than sensory encodings. This interactiveness reduces the size of the functionally activated set of alternatives. This principle is best understood in considering whether or not context cues are present during the study phase. When cues are presented only at test, as in this experiment, recall is inversely related to set size. Since there are no context cues, 
targets cannot be interactively encoded, and recall based on sensory cues will be equivalent to recall based on semantic cues for an immediate test. In constrast, when the same cues are present during both study and test phases, targets can be interactively encoded with respect to their context cues. Such interactiveness depends upon the nature of these cues. Sensory features are not interactive and, when cues and targets share letters, recall will still be an inverse function of set size. For example, given ENCH BENCH and ING SING during study, ENCH is more likely to be an effective cue since there are fewer words sharing this ending. However, semantically related cues and targets do interact, and recall does not reliably vary with set size. Continuing with an example, given Season of the Year SUMMER and Wild Animal LION during study, recall from the large taxonomic category of wild animals is just as good as recall from the small category of seasons.

According to this viewpoint, recall is a function of the interactiveness of the target with its context, the underlying principle being functional set size. Furthermore, like Craik, Nelson and McEvoy (1979) have argued that recall is a function of encoding/retrieval compatibility. However, the compatibility is defined as perceived cue-trace similarity, where similarity is identified with Tversky's (1977) contrast model. Thus, within this framework, semantic encodings activate more restricted and smaller sets. Because fewer competing alternatives are activated, semantic codes are more likely to be recalled under conditions of high levels of perceived cue-to-trace similarity.

Regardless of the disagreement expressed over the specific factors, both approaches suggest that there are at least two determinants of amount recalled on an immediate recall test, distinctiveness-compatibility or set size-similarity. The results of the present experiment suggest that decay rate might be added as a third determinant, especially when considering experiments involving relatively long delays prior to the retention test. Thus, semantic encodings may be more memorable either because they are more distinctive or because they activate smaller search sets. They also may be more memorable because they decay at a slower rate than sensory encodings. However, a caveat is appropriate. With few exceptions, the whole recent history of research on forgetting indicates that very little evidence can be found that unambiguously implicates decay as one of the causes (see Crowder, 1976, Chapters 7 and 8 for a review). Quite frankly, at the outset of the experiment we were confident that careful list construction and control of initial learning levels would result in equivalent decay rates. A clear demonstration that such was the case, even though involving acceptance of the null hypothesis, would at least suggest that decay could be disregarded as a major cause of the semantic superior- ity effect. The absence of such equivalence came as an unwelcome surprise, both for theoretical reasons and for difficulties in interpretation that such a finding provokes. These difficulties are related to possible alternative explanations that can be made when the evidence indicates that decay might be involved.

The two most obvious alternative interpretations relate to selective interference and differential rehearsal. The selective interference idea implies that the odor learning task interfered more with the visual than with the semantic components of the individual word traces. Of course, this alternative is feasible, but, nevertheless, it seems very unlikely. The use of a different modality was intentionally selected to minimize overlap with the types of information presumably being encoded during the experimental task. Thus, it would be hard to accept differential interference as the explanation for the effects of delay. The second alternative, differential rehearsal, is admittedly more plausible. In this case, if subjects rehearse during the delay, they may be more likely to rehearse semantically activated information. However, we did what we could to discourage all rehearsal. Immediately after the last slide left the screen, the experimenter engaged the subject in continuous conversation. She first indicated that this phase of the task was over, and then she walked with the subject to the next room. During this time, she tried to keep eye contact, and she essentially started instructing the subject in the nature of the odor-learning task, about the blindfold, etc. In short, when not engaged in the rather demanding odor-learning procedure, the experimenter kept each subject busy with conversation. Thus, rehearsal during the delay was certainly discouraged. However, we cannot simply dismiss the possibility.

Where do these considerations lead? To return to the data for a moment, the results of this experiment strongly suggest that visual information is encoded during study and recapitulated at test by an appropriate cue given within minutes after study. The modality effect obtained with word ending cues does not appear to be due to some sort of processing-time confounding. Modality has no effect on the redintegrative power of the taxonomic cues. The results of this experiment also indicate that delaying the retention test reduces the effectiveness of the word ending cues more than the effectiveness of the taxonomic cues. This dependency on delay could mean that visual information decays at a faster rate or that subjects are not as likely to rehearse visual information during a retention interval. This uncertainty is uncomfortable. However, two points are now clear. First, decay cannot be ruled out as a determinant of amount recalled. Second, regardless of which explanation is correct, some other determinant besides distinctiveness-compatibility or set size-similarity will have to be used to explain semantic superiority 
effects obtained on delayed retention tests. The other determinant might be either differential decay or differential rehearsal.

\section{REFERENCES}

Bregman, A. S. Forgetting curves with semantic, phonetic, graphic, and contiguity cues. Journal of Experimental Psychology, 1968, 78, 539-546.

Cermak, L. S., \& Youtz, C. P. Retention of phonemic and semantic features of words. Memory \& Cognition, 1976, 4, 172-175.

Craik, F. I. M. Overview and final comments. In L. S. Cermak \& F. I. M. Craik (Eds.), Levels of processing and human memory. Hillsdale, N.J: Erlbaum, 1979.

Craik, F. I. M., \& LockharT, R. S. Levels of processing: A framework for memory research. Journal of Verbal Learning and Verbal Behavior, 1972, 11, 671-684.

Craik, F. I. M., \& Tulving, E. Depth of processing and the retention of words in episodic memory. Journal of Experimental Psychology: General, 1975, 104, 268-294.

Crowder, R. G. Principles of learning and memory. Hillsdale, N.J: Erlbaum, 1976.

Fisher, R. P., \& Craik, F. I. M. Interaction between encoding and retrieval operations in cued recall. Journal of Experimental Psychology: Human Learning and Memory, 1977, 3, 701-711.

JACOBY, L. L. Physical features vs meaning: A difference in decay. Memory \& Cognition, 1975, 3, 247-251.

KINTSCH, W., \& BUSCHKE, H. Homophones and synonyms in short-term memory. Journal of Experimental Psychology, 1969, 80, 403-407.

Kolens, P. A. Reading a year later. Journal of Experimental Psychology: Human Learning and Memory, 1976, 2, 554-565.

Light, L. L., \& Berger, D. E. Are there long-term "literal copies" of visually presented words? Journal of Experimental Psychology: Human Learning and Memory, 1976, 2, 654-662.
Nelson, D. L. Remembering pictures and words: Appearance, significance, and name. In L. S. Cermak \& F. I. M. Craik (Eds.), Levels of processing and human memory. Hillsdale, N.J: Erlbaum, 1979.

Nelson, D. L., Brooks, D. H., \& Borden, R. C. The effects of formal similarity: Phonetic, graphic, or both? Journal of Experimental Psychology, 1974, 103, 91-96.

Nelson, D. L., \& McEvoy, C. L. Encoding context and set size. Journal of Experimental Psychology: Human Learning and Memory, 1979, 5, 292-314.

Nelson, D. L., ReEd, V. S., \& McEvoy, C. L. Learning to order pictures and words: A model of sensory and semantic encoding. Journal of Experimental Psychology: Human Learning and Memory, 1977, 3, 485-497.

Nelson, D. L., Walling, J. R., \& McEvoy, C. L. Doubts about depth. Journal of Experimental Psychology: Human Learning and Memory, 1979, 5, 24-44.

Nelson, D. L., Wheeler, J. W., Borden, R. C., \& Brooks, D. H. Levels of processing and cueing: Sensory versus meaning features. Journal of Experimental Psychology, 1974, 103, 971-977.

Shulman, H. G. Encoding and retention of semantic and phonemic information in short-term memory. Journal of Verbal Learning and Verbal Behavior, 1970, 9, 499-508.

Tulving, E. Relation between encoding specificity and levels of processing. In L. S. Cermak \& F. I. M. Craik (Eds.), Levels of processing and human memory. Hillsdale, N.J: Erlbaum, 1979.

Tversky, A. Features of similarity. Psychological Review, 1977, 84, 327-352.

UNDERWOOD, B. J. Degree of learning and the measurement of forgetting. Journal of Verbal Learning and Verbal Behavior, 1964, 3, 112-129.

(Received March 19, 1978; revision accepted April 9, 1979.) 